\title{
High SARS-CoV-2 viral load is associated with a worse clinical outcome of COVID-19 disease
}

\author{
María Eugenia Soria1,2†, Marta Cortón ${ }^{3,4}$, Brenda Martínez-González¹, Rebeca Lobo-Vega', Lucía Vázquez-Sirvent ${ }^{1}$, \\ Rosario López-Rodríguez ${ }^{3,4}$, Berta Almoguera ${ }^{3,4}$, Ignacio Mahillo5, Pablo Mínguez ${ }^{3,4}$, Antonio Herrero ${ }^{6}$, Juan \\ Carlos Taracido ${ }^{6}$, Alicia Macías-Valcayo', Jaime Esteban'1, Ricardo Fernandez-Roblas', Ignacio Gadea', Javier Ruíz- \\ Hornillos ${ }^{7,8,9, *}$, Carmen Ayuso $0^{3,4, *}$ and Celia Perales ${ }^{1,2,10, *}$
}

\begin{abstract}
COVID-19 severity and progression are determined by several host and virological factors that may influence the final outcome of SARS-CoV-2-infected patients. The objective of this work was to determine a possible association between viral load, obtained from nasopharyngeal swabs, and the severity of the infection in a cohort of 448 SARS-CoV-2-infected patients from a hospital in Madrid during the first outbreak of the pandemic in Spain. To perform this, we clinically classified patients as mild, moderate and severe COVID-19 according to a number of clinical parameters such as hospitalization requirement, need of oxygen therapy, admission to intensive care units and/or death. Also, Ct values were determined using SARS-CoV-2-specific oligonucleotides directed to ORF1ab. Here we report a statistically significant association between viral load and disease severity, a high viral load being associated with worse clinical prognosis, independently of several previously identified risk factors such as age, sex, hypertension, cardiovascular disease, diabetes, obesity and lung disease (asthma and chronic obstructive pulmonary disease). The data presented here reinforce viral load as a potential biomarker for predicting disease severity in SARS-CoV-2-infected patients. It is also an important parameter in viral evolution since it relates to the numbers and types of variant genomes present in a viral population, a potential determinant of disease progression.
\end{abstract}

\section{INTRODUCTION}

Coronavirus SARS-CoV-2 emerged in the human population in 2019 and is the causal agent of the new pandemic disease COVID-19 [1]. The virus has spread rapidly worldwide, and at the time of this writing there have been 129215179 confirmed COVID-19 cases, and 2820098 deaths worldwide, according to the WHO (https://covid19.who.int/); these numbers are increasing daily. Evolution of a virus in a specific host is defined by a number of closely related parameters, such as viral load, replication rate, genetic heterogeneity and viral fitness, that may influence virus adaptability, viral

Received 20 January 2021; Accepted 15 July 2021; Published 21 September 2021

Author affiliations: 'Department of Clinical Microbiology, Instituto de Investigación Sanitaria-Fundación Jiménez Díaz University Hospital, Universidad Autónoma de Madrid (IIS-FJD, UAM), Av. Reyes Católicos 2, 28040 Madrid, Spain; ²Centro de Biología Molecular "Severo Ochoa" (CSIC-UAM), Consejo Superior de Investigaciones Científicas (CSIC), Campus de Cantoblanco, 28049, Madrid, Spain; ${ }^{3}$ Department of Genetics \& Genomics, Instituto de Investigación Sanitaria-Fundación Jiménez Díaz University Hospital, Universidad Autónoma de Madrid (IIS-FJD, UAM), Av. Reyes Católicos 2, 28040 Madrid, Spain; ${ }^{4}$ Centre for Biomedical Network Research on Rare Diseases (CIBERER), Instituto de Salud Carlos III, 28029, Madrid, Spain; ${ }^{5}$ Department of Statistics, Instituto de Investigación Sanitaria-Fundación Jiménez Díaz University Hospital, Universidad Autónoma de Madrid (IIS-FJD, UAM), Av. Reyes Católicos 2, 28040 Madrid, Spain; 'Data Analysis Department, Instituto de Investigación Sanitaria-Fundación Jiménez Díaz University Hospital, Universidad Autónoma de Madrid (IIS-FJD, UAM), Av. Reyes Católicos 2, 28040 Madrid, Spain; ${ }^{7}$ Allergy Unit, Hospital Infanta Elena, Valdemoro, Madrid, Spain; ${ }^{8}$ Instituto de Investigación Sanitaria-Fundación Jiménez Díaz University Hospital, Universidad Autónoma de Madrid (IIS-FJD, UAM), Av. Reyes Católicos 2, 28040 Madrid, Spain; ${ }^{9}$ Faculty of Medicine, Universidad Francisco de Vitoria, Madrid, Spain; ${ }^{10}$ Centro de Investigación Biomédica en Red de Enfermedades Hepáticas y Digestivas (CIBERehd), Instituto de Salud Carlos III, 28029, Madrid, Spain.

*Correspondence: Javier Ruíz-Hornillos, javier.ruiz@quironsalud.es; Carmen Ayuso, cayuso@fjd.es; Celia Perales, celia.perales@quironsalud.es Keywords: COVID-19; risk factors; viral load.

Abbreviations: ARDS, acute respiratory distress syndrome; COPD, chronic obstructive pulmonary disease; COVID-19, coronavrus disease 2019; Ct, cycle threshold; FJD, Fundación Jiménez Díaz Hospital; HAV, hepatitis A virus; ICU, intensive care unit; ORF, open reading frame; RSV, respiratory syncytial virus; SARS-CoV-2, severe acute respiratory syndrome coronavirus-2; WHO, World Health Organization.

Associated clinical group of University Hospital Fundación Jiménez Díaz: Miguel Górgolas, Alfonso Cabello, Germán Peces Barba, Sara Heili, César Calvo, Ma Dolores Martín Ríos, Arnoldo Santos, Olga Sánchez-Pernaute, Lucía Llanos, Sandra Zazo, Federico Rojo, Felipe Villar, Raimundo de Andrés, Ignacio Jiménez Alfaro.

†These authors contributed equally to this work

$000259 \odot 2021$ The Authors 
pathogenesis and disease progression $[2,3]$. The replicative capacity of a virus is clinically relevant because it largely determines the viral load in infected individuals, and viral load influences disease manifestations [4].

In the case of SARS-CoV-2, it is a current matter of debate whether SARS-CoV-2 viral biomarkers, such as the diagnostic viral load, are able to predict progression of COVID-19 disease. A positive correlation was reported in a cohort of SARS-CoV-2-infected patients from China, showing that the viral load detected in the respiratory tract was positively linked to severity of lung disease [5]. In a related study, analysis of the viral RNA level in upper respiratory tract samples from 76 patients with COVID-19 revealed significantly lower Ct values (cycle threshold, which is inversely correlated with viral RNA level), and longer virus-shedding periods in those patients classified as severe, as compared with those who exhibited mild disease [6]. Additionally, a prospective study in a large hospitalized cohort of 1145 infected patients documented a significantly lower probability of survival in patients with high viral load than in those with low viral load [7]. Additional studies have since been published supporting the association between viral load and disease severity ([8-15], see also [16] for a review), whereas in other studies this correlation was not clear [17-22]. For example, Argyropoulos et al. documented that the diagnostic viral load level was lower in hospitalized than in non-hospitalized patients, resulting in a lack of correlation of viral load with admission to the intensive care unit (ICU), duration of oxygen support and overall patient survival [22]. Thus, the dynamics of viral load and its connection with different clinical parameters still need further characterization with additional large cohorts worldwide to define the possible association and the predictive value of viral load regarding disease progression and mortality. Our results indicate that diagnostic $\mathrm{Ct}$ values analysed from nasopharyngeal swab samples can be added to other predictive parameters to complete an early risk stratification of COVID-19 patients [23].

\section{METHODS}

\section{Patient cohort and stratification}

Data collected included patient demographics, risk factors for SARS-CoV-2 disease and clinical information related to the time of SARS-CoV-2 diagnosis (Table 1). Patients were classified according to the following COVID-19-associated parameters: (1) need of hospital admission, (2) need for mechanical ventilation, (3) admission to the ICU and (4) death attributed to COVID-19. Patients were classified as mild, moderate and severe cases according to the requirement and the type of hospitalization: (1) mild symptoms (neither hospital admission nor ICU) $(n=110),(2)$ moderate symptoms (hospitalization without ICU) $(n=236)$ and (3) severe symptoms (hospitalization with admission to the ICU, and/or death) $(n=102)$. Exceptions to these criteria are detailed in Table 1. Notably, the clinical relevance was defined before the data analysis was performed.

\section{Impact Statement}

Viral load is one of the first parameters to be measured in viral infections since it reflects the amount of virions circulating in the infected individual. Furthermore, the greater the number of virions, the greater the number of different variants present in the sample. In particular, in the new SARS-CoV-2 pandemic, there is a continuous search and urgent need for factors that could improve early prognosis of COVID-19 disease progression. Although not all studies have correlated high viral load levels in infected individuals with more severe stages of COVID-19, in our cohort we found a clear association between them. SARS-CoV-2 from nasopharyngeal swabs is the first viral sample readily available from an infected patient. Our results indicate that, in addition to their value for PCR-based diagnosis of the infection, their viral RNA load is a valid prognostic parameter for disease progression. Patients with high nasopharyngeal viral loads should be prioritized for administration of suppressive antiviral treatments.

\section{Molecular testing for SARS-CoV-2 by Ct value measurements}

Nasopharyngeal swabs were collected at Fundación Jiménez Díaz University Hospital by trained medical personnel from all patients included in the study due to suspected and then confirmed COVID-19 infection. After collection, the nasopharyngeal samples were transferred to viral transport media and transported to the Microbiology Department for molecular testing. RT-PCR to obtain diagnostic SARS-CoV-2 viral load was performed using VIASURE Real Time PCR Detection Kits by CerTest BIOTEC following the manufacturer's instructions. Ct values were calculated using SARS$\mathrm{CoV}-2$-specific oligonucleotides directed to ORF1ab.

\section{Statistics}

A normality test for the $\mathrm{Ct}$ values was performed with the Lilliefors test (Kolmogorov-Smirnov) using software $\mathrm{R}$ version 4.0.2. The statistical significance of differences among viral load values according to infection severity was calculated by ANOVA and $t$-test with Bonferroni correction using GraphPad Prism 7.00. The association between viral load and disease severity adjusted by risk factors was calculated with analysis of covariance (ANCOVA) using software R version 4.0.2.

\section{RESULTS}

Given the disparate results of correlation between viral load and COVID-19 disease progression, we addressed this question with a large cohort of 448 patients admitted to the Fundación Jiménez Díaz Hospital (FJD, Madrid, Spain) from 3 to 29 April 2020 coinciding with the first COVID-19 outbreak in Spain. At the time of admission, all patients had clinical 
Table 1. Demographic data and pre-existing comorbidities in 448 SARS-CoV-2-infected patients classified by disease severity of COVID-19

\begin{tabular}{|c|c|c|c|c|c|c|}
\hline Characteristic & $\begin{array}{c}\text { Total } \\
(n=\mathbf{4 4 8})\end{array}$ & \multicolumn{3}{|c|}{ Disease severity } & $P$-value ${ }^{\star}$ & Significance $^{\star}$ \\
\hline Age $>60$ years $(\%)$ & $321(71.6 \%)$ & $91(89.2 \%)$ & $185(78.4 \%)$ & $45(41.0 \%)$ & $1.11 \times 10^{-8}$ & $* * *$ \\
\hline Male (\%) & $205(45.7 \%)$ & $49(48.0 \%)$ & $122(51.7 \%)$ & $34(31.0 \%)$ & $1.40 \times 10^{-12}$ & $* * *$ \\
\hline Hypertension (\%) & $236(52.7 \%)$ & $69(67.6 \%)$ & $134(56.8 \%)$ & $33(30.0 \%)$ & $1.14 \times 10^{-12}$ & $* * *$ \\
\hline Cardiac disease (\%) & $142(31.7 \%)$ & $42(41.2 \%)$ & $73(30.9 \%)$ & $27(24.5 \%)$ & $1.35 \times 10^{-12}$ & $* * *$ \\
\hline Diabetes (\%) & $8(1.8 \%)$ & $2(2.0 \%)$ & $5(2.1 \%)$ & $1(0.9 \%)$ & $1.08 \times 10^{-12}$ & $* * *$ \\
\hline Hospitalization $(\%) \dagger$ & $365(81.5 \%)$ & $102(100 \%)$ & $236(100 \%)$ & $27(24.5 \%)$ & $1.21 \times 10^{-12}$ & $* * *$ \\
\hline ICU admission (\%)末 & $36(8.0 \%)$ & $33(32.3 \%)$ & $2(0.8 \%)$ & $1(0.9 \%)$ & $1.16 \times 10^{-12}$ & $* * *$ \\
\hline Conventional oxygen therapy (\%) & $288(64.3 \%)$ & $102(100 \%)$ & $183(77.5 \%)$ & $3(2.7 \%)$ & $1.10 \times 10^{-12}$ & $* * *$ \\
\hline Invasive mechanical ventilation (\%) & $19(4.2 \%)$ & $19(18.6 \%)$ & $0(0 \%)$ & $0(0 \%)$ & $1.25 \times 10^{-12}$ & $* * *$ \\
\hline Non-invasive mechanical ventilation (\%)§ & $21(4.7 \%)$ & $20(19.6 \%)$ & $1(0.4 \%)$ & $0(0 \%)$ & $1.27 \times 10^{-12}$ & $* * *$ \\
\hline High-flow nasal cannulas (\%) & $19(4.2 \%)$ & $19(18.6 \%)$ & $0(0 \%)$ & $0(0 \%)$ & $1.43 \times 10^{-12}$ & $* * *$ \\
\hline Survival 90 days after diagnosis (\%)\| & $366(81.7 \%)$ & $27(26.5 \%)$ & $233(98.7 \%)$ & $106(96.4 \%)$ & $8.44 \times 10^{-13}$ & $* * *$ \\
\hline
\end{tabular}

${ }^{*} P$-values indicate correlation between viral load and disease severity after adjusting for the baseline characteristics, pre-existing comorbilities, hospitalization, oxygen therapy and mortality. Statistical significance: ${ }^{* * *} P<0.001$; ANCOVA test.

†Exceptions are the following: the 27 patients classified as mild COVID-19 were hospitalized for causes other than SARS-CoV-2 infection. In the group moderate COVID-19, one patient was hospitalized for causes other than SARS-CoV-2 infection, but was assigned to this group due to COVID-19 pneumonia according to the clinical history.

łExceptions are the following: two patients in the group moderate COVID-19 and one patient in the group mild COVID-19 were admitted to the ICU for causes other than SARS-CoV-2 infection.

$\S$ Exceptions are the following: in the group moderate COVID-19, one patient required non-invasive mechanical ventilation during hospitalization due to a previous pathology.

IIExceptions are the following: three patients in the group moderate COVID-19 and four patients in the group mild COVID-19 died during the 90 days after diagnosis due to pathologies prior to SARS-CoV-2 infection.

IData available for 102 patients.

symptoms, and were confirmed to be positive for SARSCoV-2 by a specific real-time RT-PCR (VIASURE Real Time PCR). The Ct values analysed correspond to the first available COVID-19 PCR-positive sample for each patient. The data follow a normal distribution $[P$-value $=0.9246$; Lilliefors (Kolmogorov-Smirnov test)]. Details regarding the clinical classification are described in the Materials and Methods. Mean Ct values for mild, moderate and severe COVID-19 patients were $35.75 \pm 0.45,32.69 \pm 0.37$ and $29.58 \pm 0.70$, respectively. Univariate analysis showed statistically significant differences among viral load values according to infection severity $(P<0.0001$; ANOVA test $)$ (Fig. 1$)$. Specifically, average $\mathrm{Ct}$ values were significantly lower in the severe group as compared with the moderate and the mild disease groups, and also for the comparison between moderate and mild clinical categories $(P<0.001$ in all cases) $(t$-test with Bonferroni correction). Thus, Ct values in SARS-CoV-2-infected patients correlated positively with disease progression and poor prognosis.

Regarding host factors, it has been established that age greater than 65 years is a risk factor for developing acute respiratory distress syndrome (ARDS), a major complication of COVID-19 pneumonia, and that the risk of death increases with advanced age [24]. As a second factor, disease severity and mortality for males is significantly higher than for females, older men being the population most at risk $[25,26]$. Additionally, several co-morbidities have been potentially associated with 


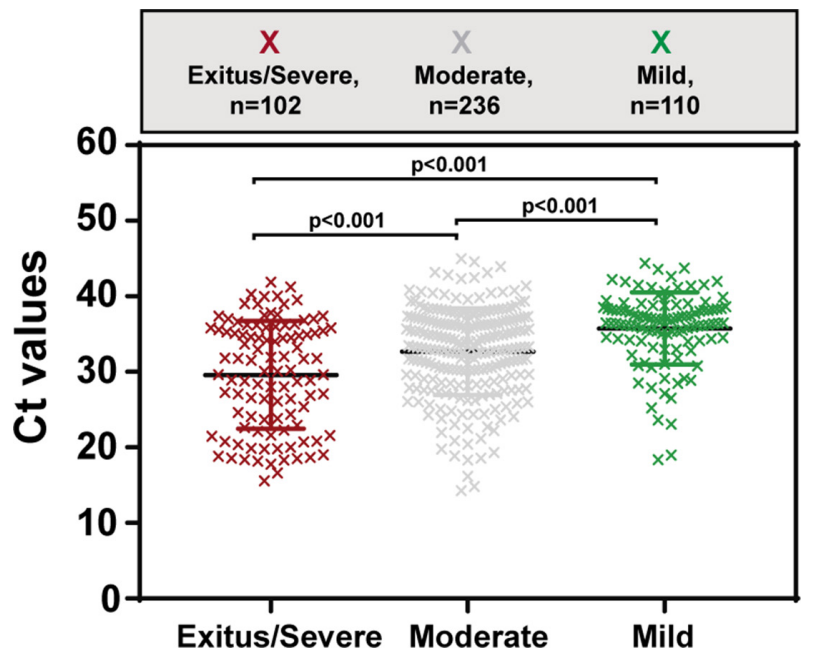

Fig. 1. Correlation between viral load [measured by the Ct (number of PCR amplification cycles needed to cross the threshold detection level) value] and the severity of COVID-19 disease.

poor outcome, including hypertension (high blood pressure), cardiovascular disease, diabetes, obesity, and lung disease (asthma, excess post-exercise oxygen consumption) [27]. We have included these risk factor data in our patient cohort to assess their alignment with our disease severity-viral RNA load association. Interestingly, a significant association between viral load and infection severity was still observed after adjusting for age, sex, hypertension, cardiovascular disease, diabetes, obesity, asthma and chronic obstructive pulmonary disease (COPD) (see ANCOVA tests in Table 1). The significant difference in viral load between the three groups was not attributable to the percentage of hospitalization, the percentage of ICU admission or the percentage of the different types of oxygen therapy (see ANCOVA tests in Table 1).

\section{DISCUSSION}

Our results with a SARS-CoV-2 population, from a cohort that is different from those in previous studies on the influence of viral load on disease, suggest a positive association between viral load and COVID-19 disease severity. This conclusion is in agreement with many studies to date [5-10, 12-15, 28, 29] (see also [16] for a review), but in contrast, others did not show such an association [17-22]. A possibility to explain such disparate conclusions is the multiple differential criteria considered among studies, such as the sample size, the genomic region analysed, the clinical severity of the patients to be compared, or the standards to classify patients in clinical categories, among other features. Our findings are in agreement with other previously published studies showing a positive correlation between viral load and COVID-19 disease. For example, a longer persistence of high viral load viruses in respiratory samples of patients with severe disease than those with mild disease has been reported, suggesting that viral load may be a prognostic parameter $[30,31]$. Ct values on admission were also predictors of COVID-19 severity in a small Japanese cohort of ICU patients $(n=19)$ whose clinical classification as 'critical' and 'non-critical' illness was based on their oxygen demand during hospitalization [28]. In a large cohort of 4254 patients in a New York City medical centre, inpatients had significantly higher $\mathrm{Ct}$ values than outpatients, and lower Ct values at admission were significantly associated with mortality [29]. In a patient cohort in Italy, SARS-CoV-2 Ct at diagnosis, within the first week of disease onset, was associated with COVID-19-related death, disease severity, number of signs and symptoms, and for the first time the presence of 6 month sequelae [15].

The time elapsed between COVID-19 onset and the diagnostic swab collection may be an important variable to consider. However, only a few studies have reported this time interval $[8,12,15]$. Our cohort includes individuals whose swab is represented by the diagnostic one, and we recorded this time interval for 102 patients. On average nasopharyngeal swabs were obtained at $7.84 \pm 6.40$ days after the onset of symptoms, which is within the time interval of active infection. It has been described that the highest SARS-CoV-2 viral load in throat swabs - and consequently the highest transmissibility peaks - is around 5-6 days after symptom onset [32], but this is an average, and values vary considerably among patients; the transmissibility window extends from a few days before symptom onset to 30 days in patients with severe disease $[30,33]$.

A correlation between high viral load and disease severity has been also found in other viral infections. Among children naturally infected with respiratory syncytial virus (RSV), increased viral load was associated with clinical severity of disease defined as an increased risk for intensive care, prolonged hospitalization or the development of respiratory failure [34]. Examination of hepatitis A virus (HAV) RNA from sera by real-time PCR resulted in higher initial viral load in patients with severe outcomes such as fulminant hepatitis and severe acute hepatitis than in patients with less severe infection [35]. This association has also been seen with other respiratory viruses such as influenza B or rhinovirus infections [36]. Viral load in patients infected with the pandemic type A influenza virus $\mathrm{H} 1 \mathrm{~N} 1$ (2009) who suffered pneumonia was higher than in patients with milder disease (those with bronchitis or upper respiratory tract infection), suggesting that viral load is also of important predictive value in influenza infection [37]. Other instances of a connection between viral load and disease progression have been reviewed [2-4].

Thus, viral load is emerging as a relevant viral parameter to predict the progression of COVID-19 and can be considered as a prognostic biomarker, together with other recognized risk factors for severity, in the management of COVID-19 patients.

\footnotetext{
Funding information

This work was supported by Instituto de Salud Carlos III, Spanish Ministry of Science and Innovation (COVID-19 Research Call COV20/00181), and co-financed by European Development Regional Fund 'A way to achieve Europe'. The work was also supported by grants CSIC-COV19-014 from Consejo Superior de Investigaciones Científicas (CSIC), BFU201791384-EXP from Ministerio de Ciencia, Innovación y Universidades
} 
(MCIU), PI18/00210 and PI21/00139 from Instituto de Salud Carlos III. C.P., M.C. and P.M. are supported by the Miguel Servet programme of the Instituto de Salud Carlos III (CPII19/00001, CPII17/00006 and CP16/00116, respectively) cofinanced by the European Regional Development Fund (ERDF). CIBERehd (Centro de Investigación en Red de Enfermedades Hepáticas y Digestivas) is funded by Instituto de Salud Carlos III. Institutional grants from the Fundación Ramón Areces and Banco Santander to the CBMSO are also acknowledged. The team at CBMSO belongs to the Global Virus Network (GVN). B.M.-G. is supported by predoctoral contract PFIS FI19/00119 from Instituto de Salud Carlos III (Ministerio de Sanidad y Consumo) cofinanced by Fondo Social Europeo (FSE). R.L.-V. is supported by predoctoral contract PEJD2019-PRE/BMD-16414 from Comunidad de Madrid. R.I-R is sponsored by the IIS-Fundación Jiménez Díaz-UAM Genomic Medicine Chair.

\section{Acknowledgements}

We acknowledge all people in the Clinical Microbiology Department of the FJD for helping with the sample and data collection. We thank all healthcare professionals who attended COVID-19 patients and collected the clinical samples that were included in this study in a difficult moment of the COVID-19 epidemic in Spain. We thank José María Aguado and Octavio Carretero for their support in the whole project. We are indebted to $E$. Domingo for encouragement and critical reading of the manuscript, and to Nuria Verdaguer and Enrique Marcos for valuable discussions about SARS-CoV-2 scientific findings.

\section{Author contributions}

C.P., I.G., R.F-R. and J.E. designed the study. A.M-V., I.G., R.F-R. and J.E. collected samples and validated RT-PCRs. M.E.S., B.M-G., R.L-V. and L.V-S. analysed Ct and clinical data. M.C., R.L-R., B.A., P.M., A.H., J.C.T., J.R-H., C.A. and C.P. classified clinically SARS-CoV-2 patients. I.M., performed statistics. J.M.A., J.R-H., C.A. and C.P. supervised analysis. C.P., M.E.S., B.M-G., R.L-V. and L.V-S. wrote the manuscript. All authors reviewed the manuscript.

\section{Conflicts of interest}

The authors declare that there are no conflicts of interest.

\section{Ethical statement}

All samples were collected according to WHO guidelines. This study was approved by the Ethics Committee and the Institutional Review Board of the FJD hospital (no. PIC-087-20-FJD). All individuals (patients) gave consent to participate in the study.

\section{References}

1. Huang $C$, Wang $Y$, Li X, Ren L, Zhao J, et al.. Clinical features of patients infected with 2019 novel coronavirus in Wuhan, China. Lancet 2020;395:497-506.

2. Domingo E, Sheldon J, Perales C. Viral quasispecies evolution. Microbiol Mol Biol Rev 2012;76:159-216.

3. DomingoE, Perales C. Viral quasispecies. PLoS Genet 2019;15:e1008271.

4. Domingo E. Virus as Populations. 2nd edn. Amsterdam: Academic Press, Elsevier; 2020.

5. Liu Y, Yang Y, Zhang C, Huang F, Wang F, et al. Clinical and biochemical indexes from 2019-nCoV infected patients linked to viral loads and lung injury. Sci China Life Sci 2020;63:364-374.

6. Liu Y, Yan L-M, Wan L, Xiang T-X, Le A, et al. Viral dynamics in mild and severe cases of COVID-19. Lancet Infect Dis 2020;20:656-657.

7. Pujadas E, Chaudhry F, McBride R, Richter F, Zhao S, et al. SARSCoV-2 viral load predicts COVID-19 mortality. Lancet Respir Med 2020;8:e70.

8. Magleby R, Westblade LF, Trzebucki A, Simon MS, Rajan M, et al. Impact of SARS-CoV-2 viral load on risk of intubation and mortality among hospitalized patients with coronavirus disease 2019. Clin Infect Dis 2020.

9. Westblade LF, Brar G, Pinheiro LC, Paidoussis D, Rajan M, et al. SARS-COV-2 viral load predicts mortality in patients with and without cancer who are hospitalized with covid-19. Cancer Cell 2020;38:661-671.

10. Faico-Filho KS, Passarelli VC, Bellei N. Is higher viral load in SARSCoV-2 associated with death? Am J Trop Med Hyg 2020;103:2019-2021.
11. Shlomai A, Ben-Zvi H, Glusman Bendersky A, Shafran N Goldberg E, et al. Nasopharyngeal viral load predicts hypoxemia and disease outcome in admitted COVID-19 patients. Crit Care 2020;24:539.

12. de la Calle C, Lalueza A, Mancheno-Losa M, Maestro-de la Calle G, Lora-Tamayo J, et al. Impact of viral load at admission on the development of respiratory failure in hospitalized patients with SARSCoV-2 infection. Eur J Clin Microbiol Infect Dis 2021;40:1209-1216.

13. Zacharioudakis IM, Prasad PJ, Zervou FN, Basu A, Inglima K, et al. Association of SARS-CoV-2 genomic load with COVID-19 patient outcomes. Ann Am Thorac Soc 2020.

14. Zacharioudakis IM, Zervou FN, Prasad PJ, Shao Y, Basu A, et al. Association of SARS-COV-2 genomic load trends with clinical status in COVID-19: A retrospective analysis from an academic hospital center in New York City. PLoS One 2020;15:e0242399.

15. Trunfio M, Venuti F, Alladio F, Longo BM, Burdino E, et al. Diagnostic SARS-COV-2 cycle threshold value predicts disease severity, survival, and six-month sequelae in COVID-19 symptomatic patients. Viruses 2021;13.

16. Rao SN, Manissero D, Steele VR, Pareja J. A systematic review of the clinical utility of cycle threshold values in the context of COVID19. Infect Dis Ther 2020;9:573-586.

17. Lesho E, Reno L, Newhart D, Clifford R, Vasylyeva O, et al. Temporal, spatial, and epidemiologic relationships of severe Acute Respiratory Syndrome Coronavirus 2 (SARS-COV-2) gene cycle thresholds: A pragmatic ambi-directional observation. Clin Infect Dis 2020.

18. Ra SH, Lim JS, Kim G-U, Kim MJ, Jung J, et al. Upper respiratory viral load in asymptomatic individuals and mildly symptomatic patients with SARS-COV-2 infection. Thorax 2021;76:61-63.

19. Jacot D, Greub G, Jaton K, Opota O. Viral load of SARS-CoV-2 across patients and compared to other respiratory viruses. Microbes Infect 2020;22:617-621.

20. Karahasan Yagci A, Sarinoglu RC, Bilgin H, Yanılmaz Ö, Sayın E, et al. Relationship of the cycle threshold values of SARS-COV-2 polymerase chain reaction and total severity score of computerized tomography in patients with COVID 19. Int J Infect Dis 2020;101:160-166.

21. Shah S, Singhal T, Davar N, Thakkar P. No correlation between Ct values and severity of disease or mortality in patients with COVID 19 disease. Indian J Med Microbiol 2021;39:116-117.

22. Argyropoulos KV, Serrano A, Hu J, Black M, Feng X, et al. Association of initial viral load in SARS-CoV-2 patients with outcome and symtoms. Am J Pathol 2020;190:1881-1887.

23. Laguna-Goya $R$, Utrero-Rico $A$, Talayero $P$, Lasa-Lazaro $M$, Ramirez-Fernandez A, et al. IL-6-based mortality risk model for hospitalized patients with COVID-19. JAllergy Clin Immunol 2020;146:799-807.

24. Ortiz-Prado E, Simbana-Rivera K, Gomez-Barreno L, Rubio-Neira M, Guaman LP, et al. Clinical, molecular, and epidemiological characterization of the SARS-CoV-2 virus and the Coronavirus Disease 2019 (COVID-19), a comprehensive literature review. Diagn Microbiol Infect Dis 2020;98:115094.

25. Guo G, Ye L, Pan $K$, Chen $Y$, Xing D, et al. New insights of emerging SARS-COV-2: Epidemiology, etiology, clinical features, clinical treatment, and prevention. Front Cell Dev Biol 2020;8:410.

26. Chen N, Zhou M, Dong X, Qu J, Gong F, et al. Epidemiological and clinical characteristics of 99 cases of 2019 novel coronavirus pneumonia in Wuhan, China: a descriptive study. Lancet 2020;395:507-513.

27. Xu J, Yang X, Yang L, Zou X, Wang Y, et al. Clinical course and predictors of 60-day mortality in 239 critically ill patients with COVID-19: a multicenter retrospective study from Wuhan, China. Crit Care 2020;24:394.

28. Fukushima $T$, Kabata $H$, Yamamoto R, Suhara $T$, Uwamino $Y$, et al. The real-time reverse transcription-polymerase chain reaction threshold cycle values for severe acute respiratory syndrome coronavirus 2 predict the prognosis of coronavirus disease 2019 pneumonia. Respir Investig 2021;59:360-363.

29. Miller EH, Zucker J, Castor D, Annavajhala MK, Sepulveda JL, et al. Pretest symptom duration and cycle threshold values for severe 
Acute Respiratory syndrome coronavirus 2 reverse-transcription polymerase chain reaction predict coronavirus disease 2019 mortality. Open Forum Infect Dis 2021;8:ofab003.

30. Zheng S, Fan J, Yu F, Feng B, Lou B, et al. Viral load dynamics and disease severity in patients infected with SARS-CoV-2 in Zhejiang province, China, January-March 2020: retrospective cohort study. BMJ 2020;369:m1443.

31. Lucas C, Wong P, Klein J, Castro TBR, Silva J, et al. Longitudinal analyses reveal immunological misfiring in severe COVID-19. Nature 2020;584:463-469.

32. He X, Lau EHY, Wu P, Deng X, Wang J, et al. Temporal dynamics in viral shedding and transmissibility of COVID-19. Nat Med 2020;26:672-675.

33. Pan Y, Zhang D, Yang P, Poon LLM, Wang Q. Viral load of SARSCoV-2 in clinical samples. Lancet Infect Dis 2020;20:411-412.
34. El Saleeby CM, Bush AJ, Harrison LM, Aitken JA, Devincenzo JP Respiratory syncytial virus load, viral dynamics, and disease severity in previously healthy naturally infected children. J Infect Dis 2011;204:996-1002.

35. Fujiwara K, Kojima H, Yasui S, Okitsu K, Yonemitsu Y, et al. Hepatitis A viral load in relation to severity of the infection. J Med Virol 2011:83:201-207.

36. Granados A, Peci A, McGeer A, Gubbay JB. Influenza and rhinovirus viral load and disease severity in upper respiratory tract infections. J Clin Virol 2017;86:14-19.

37. Li C-C, Wang L, Eng H-L, You H-L, Chang L-S, et al. Correlation of pandemic (H1N1) 2009 viral load with disease severity and prolonged viral shedding in children. Emerg Infect Dis 2010;16:1265-1272.

\section{Five reasons to publish your next article with a Microbiology Society journal}

1. The Microbiology Society is a not-for-profit organization.

2. We offer fast and rigorous peer review - average time to first decision is 4-6 weeks.

3. Our journals have a global readership with subscriptions held in research institutions around the world.

4. $80 \%$ of our authors rate our submission process as 'excellent' or 'very good'.

5. Your article will be published on an interactive journal platform with advanced metrics.

Find out more and submit your article at microbiologyresearch.org. 